\title{
Tecnologías para la comprensión lectora: estado actual y nuevos desarrollos
}

\author{
Verónica Rebolledo, Fernando Gutiérrez, Christian Soto, \\ María Fernanda Rodríguez y Diego Palma
}

\begin{abstract}
Resumen
Este artículo presenta evidencia internacional sobre el impacto de la tecnología en el rendimiento de la lectura, y describe las características centrales de algunas tecnologías que ayudan a aprender y entrenar las habilidades de comprensión lectora. Posteriormente, se exponen las particularidades de los sistemas de tutoría inteligente. Esta nueva generación de programas tiene la capacidad de recopilar datos de registro del sistema que permiten el diseño y la construcción de un modelo de estudiante más preciso, y, en muchos casos, utiliza técnicas de lenguaje natural que ayudan a generar comentarios que se adaptan mejor a las necesidades individuales de cada estudiante.
\end{abstract}

Palabras clave: ambientes virtuales de aprendizaje, comprensión de textos, sistemas tutoriales, tecnología educativa, enseñanza individualizada.

\section{TECHNOLOGIESFOR READING COMPREHENSION: CURRENTSTATUSANDNEWDEVELOPMENTS}

\begin{abstract}
This article presents international evidence on the impact of technology on reading performance, and it describes the main features of some technologies that help learn and train comprehension skills. The characteristics of intelligent tutoring systems are detailed below. This new generation of programs have the ability to collect system log data that enables the design and construction of a more accurate student model, and, in many cases, uses natural language techniques, which help to generate comments that are better adapted to the needs of each student.
\end{abstract}

Keywords: virtual learning environment, text comprehension, tutoring systems, educational technology, individualized instruction.

Recepción: 25/01/2017. Aprobación: 03/08/2020. Dol: http://doi.org/10.22201/cuaieed.16076079e.2020.21.6.7 
Verónica Rebolledo Luna

veronicarebolledoluna@gmail.com

Investigadora del área de psicolingüística, Departamento de Español, Facultad de Humanidades y Arte, Universidad de Concepción (Chile).

Fernando Gutiérrez Gómez

fernando.a.gutierrez.gomez@gmail.com

Investigador del área de psicolingüística, Departamento de Español, Facultad de Humanidades y Arte, Universidad de Concepción (Chile).

\section{Christian Soto Fajardo}

christiansotofajardo@gmail.com

Investigador del área de psicolingüística, Departamento de Español, Facultad de Humanidades y Arte, Universidad de Concepción (Chile).

\section{María Fernanda Rodríguez Poblete}

mfernanda.rodriguez16@gmail.com

Investigadora del área de psicolingüística, Departamento de Español, Facultad de Humanidades y Arte, Universidad de Concepción (Chile).

\section{Diego Palma Sánchez}

dpalmasan@gmail.com

Investigador del área de psicolingüística, Departamento de Español, Facultad de Humanidades y Arte, Universidad de Concepción (Chile). 


\section{Introducción}

La comprensión lectora es una de las habilidades más importantes en el contexto escolar, ya que muchas actividades intelectuales que los estudiantes deben realizar, tanto en el colegio como fuera de éste, suponen un óptimo desarrollo de estas competencias.

Desafortunadamente, las pruebas de lectura muestran que en Latinoamérica los estudiantes no logran los resultados esperados y, en consecuencia, esto impacta negativamente la forma en que aprovechan las oportunidades de aprendizaje escolar (OCDE, 2014).

Por lo anterior, la enseñanza de este tipo de habilidades no resulta una tarea sencilla, pues los educadores deben poseer conocimiento especializado sobre los procesos psicológicos de la comprensión, además de un conocimiento didáctico sobre cómo enseñar este tipo de estrategias, que habitualmente no son parte del bagaje pedagógico del profesor.

Los docentes requieren de herramientas computacionales que ofrezcan retroalimentación individualizada, y que también capturen las diferencias individuales de cada sujeto, puesto que existe una gran diversidad de estudiantes que necesitan apoyos diferenciados. Debido a ello, hay que fomentar nuevas formas de acompañamiento, que consoliden este tipo de aprendizaje mediante actividades prácticas y automatizadas, aplicables en el aula.

\section{Algunas evidencias sobre el uso de tecnologías y el desempeño lector}

San Martín, Jara, Preiss, Claro y Fariña (2012) realizaron una investigación que intentó valorar los aportes de las Tecnologías de la Información y la Comunicación (TIC) en los resultados PISA de lectura en Chile, Uruguay, España, Portugal y Suecia. Este estudio examinó los factores específicos que incidían en el desempeño lector de los estudiantes de países con diferente nivel de desarrollo.

Uno de los hallazgos más interesantes fue que el factor tıc tuvo diferente peso sobre el desempeño en la prueba de lectura de la evaluación PISA, de acuerdo al país que se considere. En los países sudamericanos evaluados, la actividad de lectura en línea mostró contribuir a mejorar los rendimientos, lo que se traduce en una mayor influencia de la lectura en línea en dichos países, comparada con países como España y Portugal.

Las investigaciones parecen confirmar que cuando existen menores niveles de desempeño lector, la lectura a través de un computador (de diferente tipo de texto) ayuda a incrementar los rendimientos (San Martín et al., 2012; Johnson, Jacovina, Russell y Soto, 2016). Ello se debería a que el uso de contenidos en internet, que son presentados principalmente como texto escrito, promueve que los estudiantes lean más de lo que harían sin el computador. 
Las conclusiones de Jackson, Von Eye, Biocca, Barbatsis, Zhao y Fitzgerald (2006) muestran hallazgos similares con estudiantes de Estados Unidos. Así, en cierta forma, se ratifica lo que planteó la oCDE en el año 2010, cuando afirmó que los estudiantes que participan con frecuencia en actividades de lectura en línea son, en general, lectores más competentes.

Adicionalmente, cuando los estudiantes utilizan tecnología para realizar actividades online, entrenan sus habilidades lectoras a través de un soporte que resulta ser más atractivo, porque se suma el peso de factores motivacionales como una variable fundamental en el proceso lector (Crossley y McNamara, 2016).

Por tal razón, agregar tiempos de lectura mediante algún aparato tecnológico podría ser un primer camino para que los educandos practiquen sus habilidades de lectura, puesto que los estudiantes manifiestan una disposición y compromiso superiores con respecto a las prácticas tradicionales de lectura sobre papel.

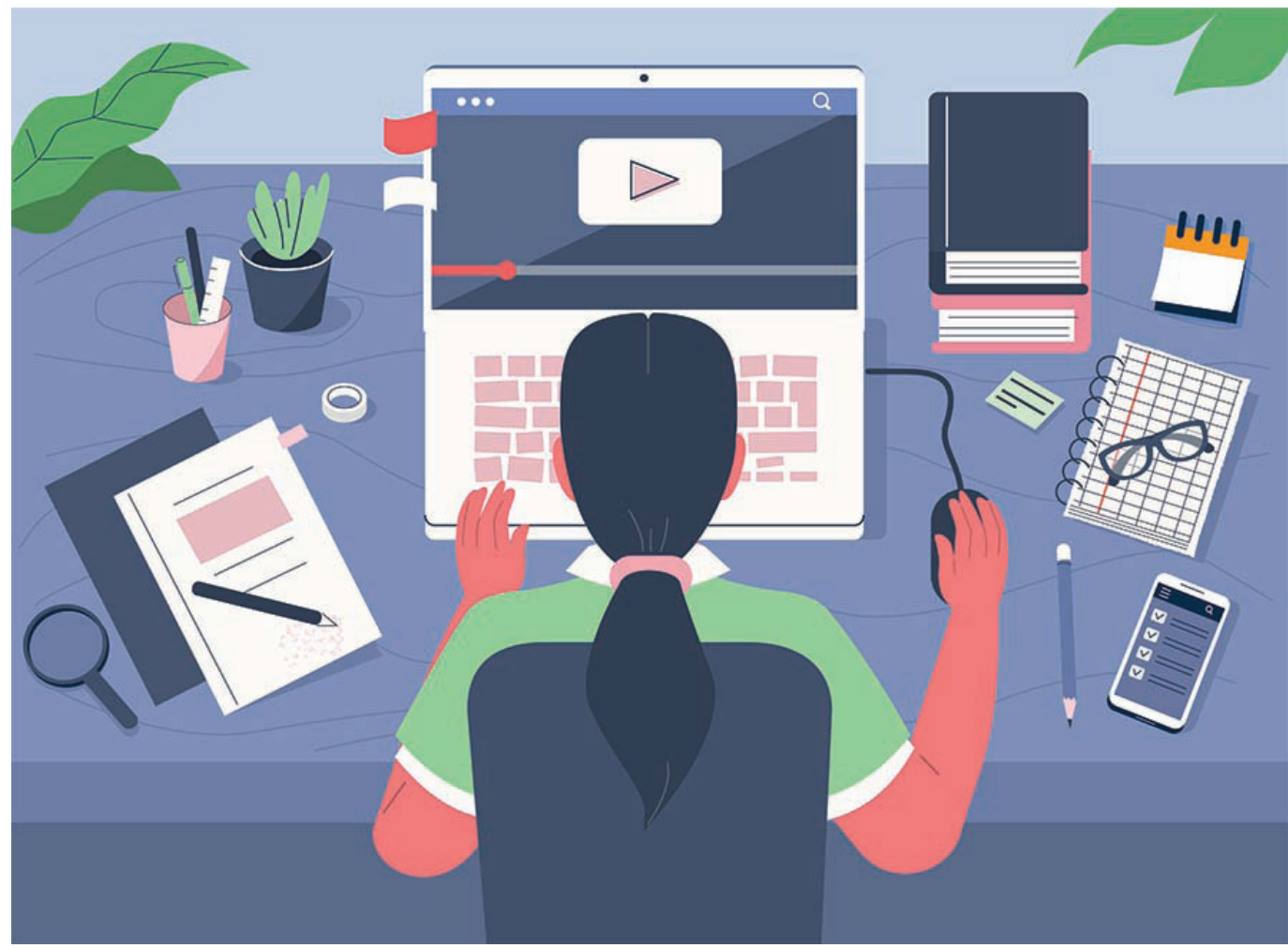

\section{Herramientas computacionales que ayudan a docentes y estudiantes en la comprensión de textos}

Al generarse más oportunidades de lectura, los estudiantes activan y entrenan sus habilidades; sin embargo, el problema surge cuando su repertorio de destrezas es muy escaso. Incorporar nuevas estrategias supone un proceso en el que los estudiantes deben aprender e implementar nuevas formas de aproximarse al texto, en busca de un procesamiento más profundo al utilizar estrategias de comprensión. 
En este contexto, comienzan a surgir nuevas herramientas computacionales para apoyar el aprendizaje de las estrategias de comprensión que ayudan a los docentes y a una gran diversidad de alumnos a entrenar estas habilidades tan fundamentales para el currículo escolar.

La tecnología educativa enfocada en comprensión lectora que posee la mayor cantidad de estudios y publicaciones, que dan cuenta de su eficacia, es isTART (Interactive Strategies Training for Active Reading and Thinking) creada por Danielle McNamara y su equipo (McNamara, O'Reilly, Rowe, Boonthum y Levinstein, 2007; Snow, Jacovina, Jackson, y McNamara, 2016). El objetivo de isTART es mejorar la comprensión lectora de estudiantes de secundaria a través de la enseñanza y entrenamiento de cinco estrategias: monitoreo, puente, predicción, elaboración y parafraseo. Los estudiantes deben leer partes de textos científicos y luego elaborar autoexplicaciones acerca de esas piezas de información, utilizando alguna de las estrategias aprendidas. Este programa también ha sido adaptado al español en su versión llamada isTART-E. Dicha versión fue probada en estudiantes de Argentina y Chile, y los resultados en ambos estudios demuestran una mejora significativa en el desempeño en comprensión lectora de los participantes (McCarthy, Soto, Malibran, Fonesca, Simian y McNamara, 2018).

Dentro de los programas computacionales disponibles en español también se destacan tuinLEC y COMPRENDE. TuinıEC (Vidal-Abarca et al., 2014) está dirigido a estudiantes españoles de último curso de educación primaria y primer curso de secundaria. Busca mejorar la competencia lectora al enseñar cuatro estrategias enfocadas en: 1) cómo leer textos continuos y discontinuos, 2) cómo entender y responder preguntas, 3) cuándo decidir releer el texto para responder, y 4) cómo buscar en el texto para responder. El entrenamiento incluye de seis a ocho preguntas por texto, las cuales siguen los lineamientos de la prueba PISA. Por otro lado, comprende (Soto, Gutiérrez de Blume, Rodríguez, Asún, Figueroa, y Serrano, 2019) es una tecnología educativa diseñada para enseñar y entrenar estrategias de comprensión lectora en alumnos de $5^{\circ}$ a $7^{\circ}$ año de primaria. Para ello cuenta con dos fases: Lecciones y Entrenamiento. En la fase de Lecciones, un agente animado enseña a aplicar las estrategias de vocabulario contextual, inferencias puente, integración de ideas y comprensión global. Posteriormente, en la fase de Entrenamiento, el estudiante debe ejecutar diferentes tareas como responder preguntas de selección múltiple y abiertas, elaborar resúmenes de lo que se leyó, entre otras, mientras recibe retroalimentaciones, con enfoque metacognitivo, por parte del agente animado del programa. Las pruebas de validación de COMPRENDE evidencian que el programa es efectivo en la mayoría de los participantes; sin embargo, los alumnos que más se beneficiaron son los que presentan un desempeño lector inicial más bajo (Soto et al., 2019).

Los diversos programas que apoyan la comprensión actúan sobre el incremento de las oportunidades de lectura a través de prácticas recurrentes, al mismo tiempo que enseñan intencionadamente estrategias para que el estudiante mejore el nivel de procesamiento de los textos. No obstante, aún se 
requiere que este tipo de programas se focalice en las diferencias individuales de cada sujeto, para que estudiantes con alguna necesidad educativa especial (NEE), como el trastorno del espectro autista (TEA), puedan beneficiarse y desarrollar habilidades de comprensión. En este sentido, una propuesta innovadora es la presentada por el programa YaLeo (Rodríguez y Soto, 2020), cuyo contenido apunta a mejorar la comprensión lectora al atender a las necesidades específicas de la población con neE, tales como la enseñanza explícita de estrategias, apoyos visuales y uso del modelado. Por otra parte, la complejidad de los textos presentados va aumentando en forma paulatina: desde la comprensión de oraciones, textos narrativos, hasta el abordaje de textos expositivos.

Hasta el momento nos hemos enfocado en el importante peso de las estrategias sobre la mejora en la comprensión, al destacar la utilidad que podrían tener los métodos de entrenamiento automatizados. No obstante, no queremos terminar esta sección sin antes mencionar la importancia que tienen en este proceso las características del texto. Más allá de la diversidad de los géneros discursivos, la complejidad del texto en sí mismo puede darnos pistas importantes sobre la naturaleza de la dificultad con la que se encontrará el lector. Por tanto, es relevante determinar qué hace más complejo a un texto en una circunstancia determinada, y qué combinación de variables del texto debieran ser fundamentales para definir que es adecuado para el trabajo de escolares en diversos niveles educativos. Al utilizar indicadores lingüísticos del texto, tales como sus características sintácticas y semánticas, e integrarlos con la experiencia del docente, se ha demostrado que los índices relevantes en la comprensión lectora variarán dependiendo de los variados contextos que se hayan determinado (Palma y Soto, 2020). Por otro lado, para promover las investigaciones en torno a la complejidad de textos en español, es importante contar con herramientas y/o bibliotecas computacionales que permitan compartir criterios lingüísticos definidos, que sean posibles de reproducir por diversos profesionales e investigadores, como la biblioteca de código abierto TRUNAJOD ${ }^{1}$ para análisis de complejidad textual.

\section{Características fundamentales de las tecnologías que impactan sobre el aprendizaje de la comprensión}

Las actuales tendencias en tecnologías para la comprensión giran en torno a programas que se perfeccionan en torno a versiones cada vez más complejas, incorporando nuevos desarrollos o ajustes específcos. Estas innovaciones permiten mejorar la eficacia, al mismo tiempo que posibilitan nuevos caminos para la investigación, como ocurre con isTART, ISTART-ME o isTART 2. Jackson y McNamara (2013) plantean las ventajas de integrar inteligencia artificial y juegos a estos sistemas automatizados para incrementar la motivación de los estudiantes en torno a las tareas a realizar.

En la actualidad se está estudiando cómo el juego ayuda a mantener la motivación, el compromiso y la disposición del estudiante para proseguir con 
Cuadro 1. Algunas características básicas que demuestran ser útiles en el diseño y uso de tecnologías para la comprensión.

https://github.com/dpalmasan/ TRUNAJOD2.0 las tareas de aprendizaje. Por otro lado, un buen diseño de las actividades del programa, feedback significativos y refuerzos específicos, inciden sustancialmente en la disposición del estudiante para avanzar al siguiente paso en la adquisición de las habilidades.

\begin{tabular}{|c|c|}
\hline $\begin{array}{c}\text { Foco en } \\
\text { estrategias }\end{array}$ & $\begin{array}{l}\text { - Los programas incluyen técnicas o procedimientos que ayudan al } \\
\text { lector a implementar nuevas operaciones que tienden a establecer } \\
\text { mejores conexiones en torno a las ideas del texto. } \\
\text { - El aprendizaje de estrategias parece ser un pilar central en los } \\
\text { programas de comprensión y resulta, por tanto, natural incorporarlo. } \\
\text { Presentan una primera fase de lecciones centradas en presentar } \\
\text { un número limitado de estrategias, en que el programa enseña a } \\
\text { utilizarlas, para luego entrenarlas en una fase posterior del proceso } \\
\text { (Snow et al., 2016). }\end{array}$ \\
\hline $\begin{array}{l}\text { Variados } \\
\text { recursos y } \\
\text { retroalimentación }\end{array}$ & $\begin{array}{l}\text { - Los programas ofrecen diferentes recursos para el estudiante que, } \\
\text { en su conjunto, ayudarán a fortalecer y reforzar las habilidades de } \\
\text { comprensión que han sido enseñadas previamente. } \\
\text { - La retroalimentación y los refuerzos pueden ayudar a generar andamios } \\
\text { relevantes, que direccionan los procesos de aprendizaje alcanzados, } \\
\text { por lo que su buen diseño puede ayudar a mejorar el impacto de toda } \\
\text { la herramienta (Gutiérrez y Atkinson, 2011). }\end{array}$ \\
\hline $\begin{array}{c}\text { Enfoque } \\
\text { personalizado }\end{array}$ & $\begin{array}{l}\text { - Una tecnología que se adapte a las características individuales ayudará } \\
\text { a estudiantes con y sin NeE a mantener la motivación, y a que los } \\
\text { recursos del programa sean más efectivos para cada sujeto. } \\
\text { - Registrar el recorrido individual del sujeto ayuda a generar } \\
\text { investigaciones que detecten variables y comportamientos críticos } \\
\text { que están operando durante el uso del sistema (Crossley y McNamara, } \\
\text { 2016). }\end{array}$ \\
\hline $\begin{array}{l}\text { Considera la } \\
\text { motivación }\end{array}$ & $\begin{array}{l}\text { - La motivación incluye la disposición emocional y conductual de los } \\
\text { estudiantes para animarse a utilizar este tipo de programas, continuar } \\
\text { trabajando en el sistema e, incluso, aumentar la frecuencia con que lo } \\
\text { utilizan. } \\
\text { - La actual tendencia es incorporar minijuegos en los programas } \\
\text { computacionales o generar ambientes que simulen juegos } \\
\text { computacionales (isTART, LECTOR-Código UdeC, comPRENDE). }\end{array}$ \\
\hline
\end{tabular}

\section{Características que tendrán los futuros desarrollos en tecnologías para la comprensión}

Uno de los caminos innovadores en torno a estos programas lo ofrecen los sistemas tutoriales inteligentes (STI). En general, estos sistemas son ambientes de aprendizaje basados en computadoras, que proporcionan a los estudiantes instrucción y práctica. Los stı han sido desarrollados para una amplia variedad de temas, que van desde la física a la comprensión de lectura (Graesser, McNamara, y VanLehn, 2005).

El desarrollo de los stı ha sido influido por numerosas disciplinas, tales como las ciencias del aprendizaje, la psicología, la informática, la lingüística y la inteligencia artificial. stı bien diseñados han demostrado ganancias en el aprendizaje similares a las generadas por tutores humanos, lo que demuestra su gran potencial (VanLehn, 2011). 
Se consideran inteligentes porque adaptan su entrenamiento y retroalimentación de acuerdo con los desempeños individuales de los estudiantes. Esta adaptación funciona a través del registro de la información de los alumnos, ya que el sistema utiliza dichos datos para influir en el proceso mismo de entrenamiento.

Los investigadores pueden tener razones teóricas para predecir que ciertos comportamientos se relacionan, por ejemplo, con alguna NEE, pero a menudo es necesario confirmar estas predicciones preguntando explícitamente a los sujetos, observando cómo usan el programa o tomando en cuenta otros comportamientos relacionados (Baker, D'Mello, Rodrigo, y Graesser, 2010). Todo este tipo de información en el sistema genera una especie de representación de las características del sujeto, al que se le denomina modelo del estudiante VanLehn, 2006). Éste puede ser actualizado para reflejar los comportamientos sensibles que requieren ser identificados por el programa. El modelo del estudiante permite adaptar las propiedades y recursos del sistema de acuerdo con las características individuales de cada sujeto, contribuyendo así a un enfoque inclusivo.

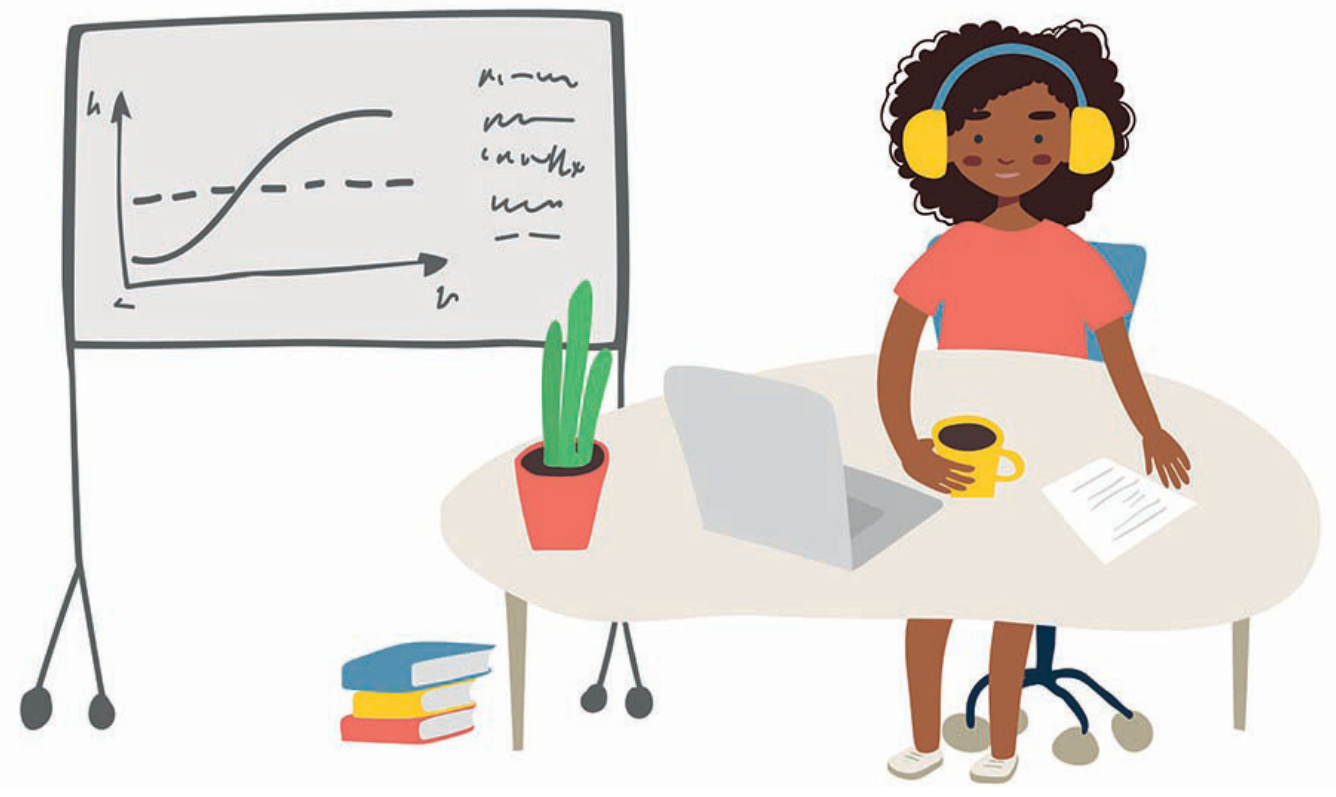

\section{Otros componentes de los stı para la comprensión lectora}

Cabe destacar tres componentes en los stı que están jugando un rol importante en los desarrollos en tecnologías para la compresión lectora. El primero corresponde al procesamiento del lenguaje natural (PLN), que basa su funcionamiento en el análisis automatizado de la producción, ya sea oral o escrita, del sujeto. Como señalan McNamara y su equipo (2007), estos algoritmos pueden proporcionar evaluaciones sumativas o formativas del trabajo escrito de los estudiantes, lo que responde a un análisis más adecuado para capturar diferencias individuales. Estos sistemas incorporan técnicas tan variadas como 
representación de conocimiento (Gutiérrez, Dou, Fickas y Griffiths, 2012) o conjuntos de textos (Foltz, Laham y Landauer, 1999) para poder determinar el contenido de las repuestas escritas de los estudiantes.

Al enfocarse en el contenido de las respuestas escritas, estas nuevas técnicas de análisis de textos pueden determinar si una oración esta fuera del contexto de la pregunta o si tiene errores lógicos. Aunque estos algoritmos no son perfectos, animan a los estudiantes a participar activamente en la generación de respuestas escritas, porque les entregan retroalimentación directa relacionada con dichas respuestas.

El segundo componente de los sti que se debe mencionar es la retroalimentación, la cual es crucial ya que proporciona información útil para que los estudiantes dominen con éxito las estrategias de comprensión lectora que están practicando (Ericsson, 2008). Han surgido nuevos desarrollos en el tema de la retroalimentación, lo que augura que a futuro los feedback serán cada vez más precisos. Por ejemplo, Gutiérrez y Atkinson (2011) han propuesto un método adaptativo basado en técnicas de inteligencia artificial, que integra las respuestas previas del estudiante y el tipo de error que ha cometido. Este método permite mejorar la estrategia de retroalimentación e induce al estudiante a ser más preciso en su respuesta y a cometer menos errores.

Finalmente, una manera cada vez más típica para mejorar la motivación es el uso de juegos y características similares al juego en todo el entorno de aprendizaje (Gee, 2005). Un buen desempeño en las actividades educativas puede ser recompensado con puntos, que permitan al estudiante jugar minijuegos o personalizar un avatar (Jackson y McNamara, 2013), lo que inducirá a comprometerse de mejor manera con la tarea.

\section{Conclusiones}

En los últimos años se han desarrollado programas específicos que enseñan y entrenan habilidades de comprensión lectora y que están demostrando interesantes evidencias de impacto positivo en la mejora del desempeño lector.

Los futuros desarrollos en este tipo de tecnologías requieren incorporar nuevos avances en inteligencia artificial para posibilitar una mejor adaptación del programa a las necesidades y características individuales del usuario (modelo del estudiante), generando así una respuesta educativa a la diversidad desde un enfoque inclusivo.

Dado que existe una apremiante necesidad de mejorar los desempeños en lectura en los países latinoamericanos, el uso de tecnologías para su comprensión, surge como una herramienta de apoyo muy prometedora para la educación en general; particularmente en el acompañamiento de escolares con necesidades educativas especiales, como los estudiantes con trastorno del espectro autista. 
Los nuevos desarrollos en estas tecnologías ayudarán a obtener mejores resultados al generar herramientas de apoyo cada vez más eficaces en la tarea de potenciar una habilidad tan importante y compleja como es la comprensión lectora.

Agradecimiento: Este trabajo recibió el apoyo del proyecto FONDEF ID 20110290: "Literador: Un tutor inteligente que potencia las competencias en lectura y escritura" (ANID, Chile).

\section{Referencias}

* Baker, R., D'Mello, S, Rodrigo, M. y Graesser, A. (2010). Better to be frustrated than bored: The incidence, persistence, and impact of learners' cognitiveaffective states during interactions with three different computer-based learning environments. International Journal of Human-Computer Studies, 68,(4) 223-241. Dol: https://doi.org/10.1016/j.ijhcs.2009.12.003

* Crossley, S. y McNamara, D. (2016). Adaptive educational technologies for literacy instruction. Taylor \& Francis.

* Ericsson, K. (2008). Deliberate practice and acquisition of expert performance: A general overview. Academic Emergency Medicine, 15(11), 988-994. Dol: https://doi. org/10.1111/j.1553-2712.2008.00227.x

* Foltz, P., Laham, D., y Landauer, T. (1999). Automated essay scoring: Applications to educational technology. En B. Collis y R. Oliver (Eds.), Proceedings of EDMedia '99 (pp. 939-944).

* Gee, J. (2005). Why video games are good for your soul: Pleasure and learning. Common Ground Press.

* Graesser, A., Mcnamara, D. y VanLehn, K. (2005). Scaffolding deep comprehension strategies through point \& query, AutoTutor, and istart. Educational Psychologist, 40(4), 225-234. Dol: https://doi.org/10.1207/s15326985ep4004_4

* Gutiérrez, F. y Atkinson, J. (2011). Adaptive feedback selection for intelligent tutoring systems. Expert Systems with Applications, 38(5), 6146-6152. Dol: https:// doi.org/10.1016/j.eswa.2010.11.058

* Gutiérrez, F., Dou, D., Fickas, S. y Griffiths G. (2012). Providing grades and feedback for student summaries by ontology-based information extraction. Proceedings of the 21st ACM International Conference on Information and Knowledge Management, 1722-1726. Dol: https://www.doi.org/10.1145/2396761.2398505

- Jackson, G. y McNamara, D. (2013). Motivation and performance in a game-based intelligent tutoring system. Journal of Educational Psychology, 105(4), 1036-1049. Dol: https://doi.org/10.1037/a0032580

- Jackson, L. A., von Eye, A., Biocca, F. A., Barbatsis, G., Zhao, Y. y Fitzgerald, H. E. (2006). Does home internet use influence the academic performance of lowincome children? Developmental Psychology, 42(3), 429-435. Dol: https://doi. org/10.1037/0012-1649.42.3.429 
* Johnson, A., Jacovina, M., Rusell, D. y Soto, C. M. (2016). Challenges and solutions when using technologies in the classroom. En D. S. McNamara y S. A. Crossley (Eds.), Adaptive educational technologies for literacy instruction (pp. 13-29). Taylor \& Francis, Routledge.

* McCarthy, K. S., Soto, C., de Blume, A. P. G., Palma, D., González, J., y McNamara, D. Improving reading comprehension in Spanish using istART-E: A pilot study. International Journal of Computer-Assisted Language Learning and Teaching (IJCALLT; ISSN: 2155-7101), Special Issue: WorldCALL 2018: CALLing all the CALLers Worldwide. https://www.researchgate.net/profile/Antonio_Gutierrez_De_Blume/ publication/340952008_Improving_Reading_Comprehension_in_Spanish_using_ iSTART-E_A_Pilot_Study/links/5ea7117e299bf1125612f51b/Improving-ReadingComprehension-in-Spanish-using-iSTART-E-A-Pilot-

* McCarthy, K. S., Soto, C., Malbrán, C., Fonseca, L., Simian, M., y McNamara, D. S. (2018). istART-E: Reading comprehension strategy training for Spanish speakers. En C. P. Rosé, R. Martinez-Maldonado, U. Hoppe, R. Luckin, M. Mavrikis, K. PorayskaPomsta, B. McLaren y B. D. Boulay (Eds.), Artificial Intelligence in Education [19th International Conference, AIED 2018, London, UK, June 27-30, 2018, Proceedings, Part II], pp. 215-219. Springer.

* McNamara, D., O'Reilly, T., Rowe, M., Boonthum, C. y Levinstein, I.B. (2007). istarT: A web-based tutor that teaches self-explanation and metacognitive reading strategies. En D.S. McNamara (Ed.), Reading comprehension strategies: Theories, interventions, and technologies (pp. 397-420). Erlbaum.

* McNamara, D., Boonthum, C., Levinstein, I. y Millis, K. (2007). Evaluating selfexplanations in isTART: Comparing word-based and LSA algorithms. En T. K. Landauer, D. S. McNamara, S. Dennis, y W. Kintsch (Eds.), Handbook of Latent Semantic Analysis (pp. 227-241). Erlbaum.

- OECD. (2010). PISA 2009 Results: Learning to Learn-Student Engagement, Strategies and Practices (Volume III). Dol: http://dx.doi.org/10.1787/9789264083943-en

- OECD. (2014). PISA 2012 Results: What Students Know and Can Do - Student Performance in Mathematics, Reading and Science (Volume I, REVISED EDITION, FEBRUARY 2014), PISA, oeCD Publishing. Dol: http://dx.doi.org/10.1787/9789264201118-en

* Palma, D., Soto, C., Veliz, M., Riffo, B., y Gutiérrez, A. (2019, agosto). A datadriven methodology to assess text complexity based on syntactic and semantic measurements. International Conference on Human Interaction and Emerging Technologies, 509-515 Springer, Cham.

* Palma, D., Soto, C., Veliz, M., Riffo, B., Gutiérrez, A. (2020). A Data-Driven Methodology to Assess Text Complexity Based on Syntactic and Semantic Measurements. En Ahram T., Taiar R., Colson S., Choplin A. (Eds.), Human Interaction and Emerging Technologies. ihiet 2019. Advances in Intelligent Systems and Computing (Vol. 1018). Springer, Cham. Dol: https://doi.org/10.1007/978-3-030-25629-6_79

* Rodríguez, F. y Soto, C. (2020). YaLeo: programa para mejorar el desempeño lector en estudiantes con Necesidades Educativas Especiales [ANID, ProyeCto fondef VIU18E0088]. 
* San Martín, E., Jara, I., Preiss, D., Claro, M. y Fariña, P. (2012). ¿Cuán relevante es el aporte de diversos usos de TIC para explicar el rendimiento lector en PISA? Modelando el aporte neto tic en Chile, Uruguay, España, Portugaly Suecia [informe de investigación Proyecto Fonide N: FE11124 MINEDUc, Chile].

- Snow, E., Jacovina, M., Jackson, G.T., y McNamara, D. (2016). istart-2. A reading comprehension and strategy instruction tutor. En D. S. McNamara y S. A. Crossley (Eds.), Adaptive educational technologies for literacy instruction (pp. 104-121). Taylor \& Francis, Routledge.

* Soto, C., de Blume, A. P. G., Rodríguez, M. F., Asún, R., Figueroa, M., y Serrano, M. (2019). Impact of bridging strategy and feeling of knowing judgments on reading comprehension using COMPRENDE: An educational technology. TechTrends, 63(5), 570-582. Dol: http://dx.doi.org/10.1007/s11528-019-00383-5

* VanLehn, K. (2006). The behavior of tutoring systems. International Journal of Artificial Intelligence in Education, 16(3), 227-265.

* VanLehn, K. (2011). The relative effectiveness of human tutoring, intelligent tutoring systems, and other tutoring systems. Educational Psychologist, 46(4), 197-221. Dol: https://doi.org/10.1080/00461520.2011.611369

* Vidal-Abarca, E., Gilabert R., Ferrer, A., Avila V., Martínez, T., Maña, A., Llorens A., Gil, L., Cerdán R., Ramos, L. y Serrano, M. (2014). TuinıEc, un tutor inteligente para mejorar la competencia lectora. Journal for the Study of Education and Development, Infancia y Aprendizaje, 37(1), 25-56.

\section{Cómo CITAR ESTE ARTículo}

* Rebolledo, Verónica, Gutiérrez, Fernando, Soto, Christian, Rodríguez, María Fernanda y Palma, Diego. (2020, noviembre-diciembre). Tecnologías para la comprensión lectora: estado actualy nuevos desarrollos. Revista Digital Universitaria (RDU), 21(6). Dol: http://doi.org/10.22201/cuaieed.16076079e.2020.21.6.7 